\title{
Polycistronic expression of RNA silencing suppressor protects its own mRNA from RNA silencing
}

\author{
Yukio Kurihara, Emiko Okubo-Kurihara, Minami Matsui* \\ Synthetic Genomics Research Team, Biomass Engineering Program Cooperation Division, RIKEN Center for Sustainable \\ Resource Science, Yokohama, Kanagawa 230-0045, Japan \\ *E-mail: minami@riken.jp Tel: +81-45-503-9625 Fax: +81-45-503-9586
}

Received October 2, 2014; accepted January 20, 2015 (Edited by Y. Chiba)

\begin{abstract}
The internal ribosome entry site (IRES) enables polycistronic expression of multiple genes from a single mRNA controlled by one promoter. In general, only the most 5' coding sequence is abundantly translated from polycistronic mRNAs in eukaryotic cells; IRES-mediated translation allows additional coding sequences at other positions to be translated at detectable levels. However, IRES-mediated translation often results in much lower protein expression than translation of single-gene mRNAs. We first aimed to improve IRES-mediated gene expression with a transient expression system based on Nicotiana benthamiana. We demonstrated that the presence of two cassettes comprised of an IRES-mediated Venus coding sequence results in higher Venus expression than one cassette. The double IRES cassette system is expected to be useful for expressing a gene of interest polycistronically in plants. Using the double IRES cassette system, we found that polycistronic expression of a reporter gene and two copies of an IRES-mediated RNA silencing suppressor gene protected the transcribed mRNA from RNA silencing-mediated degradation. This is the first report of a mRNA self-protection system from RNA silencing by IRES-mediated expression of a viral suppressor in plants.
\end{abstract}

Key words: IRES, RNA silencing, p19.

In eukaryotes, a single endogenous mRNA is generally monocistronically translated into one protein in a capdependent manner. However, the viral internal ribosome entry site (IRES) recruits ribosomes to the mRNAs that contain them and facilitates internal translation to produce viral proteins in a cap-independent manner (Thompson 2012). IRESs were first discovered in animal viruses (Jang et al. 1988; Pelletier and Sonenberg 1988) and have since been identified in some plant RNA viruses.

IRESs have been incorporated into virus vectors to facilitate polycistronic expression of multiple genes in eukaryotic cells (Thompson 2012). In particular, an IRES element derived from the coat protein gene of the crucifer Tobacco Mosaic Virus (cr-TMV) has been used for polycistronic gene expression in plants (Ha et al. 2010; Skulachev et al. 1999). Yamamoto et al used the cr-TMV IRES to selectively express a firefly luciferase gene from a single mRNA that also encoded a Renilla luciferase gene positioned $5^{\prime}$ to the firefly gene (Yamamoto et al. 2003).

RNA silencing, also referred to as RNA interference, involves post-transcriptional degradation of RNAs mediated by short (21-24nt) interfering RNAs (siRNAs) in a sequence-dependent manner (Baulcombe 2004). RNA silencing is a host defense system against invading viruses (Pumplin and Voinnet 2013). To defeat this defense system, many plant viruses encode RNA silencing suppressors (Voinnet et al. 1999). The p19 protein of Tombusvirus, an RNA silencing suppressor that is widely used in transgenic experiments, binds viral siRNAs and abrogates siRNA-mediated degradation of viral RNAs (Vargason et al. 2003). Until now, the suppressor activity of the p19 protein has been used to maintain expression of genes by co-expression of $\mathrm{p} 19$ as a separate mRNA (Voinnet et al. 2003).

In this report, we first attempted to improve IRESmediated polycistronic expression by a transient expression system using Nicotiana benthamiana. In addition, we established a novel mRNA self-protection system from RNA silencing using two IRES cassettes harboring the $p 19$ gene.

Abbreviations: IRES, internal ribosome entry site; cr-TMV, crucifer Tobacco Mosaic Virus; PYR1, PYRABACTIN RESISTANCE 1; p19, Tombusvirus $19 \mathrm{kDa}$ protein; HPT, hygromycin phosphotransferase.

This article can be found at http://www.jspcmb.jp/

Published online February 24, 2015 
A

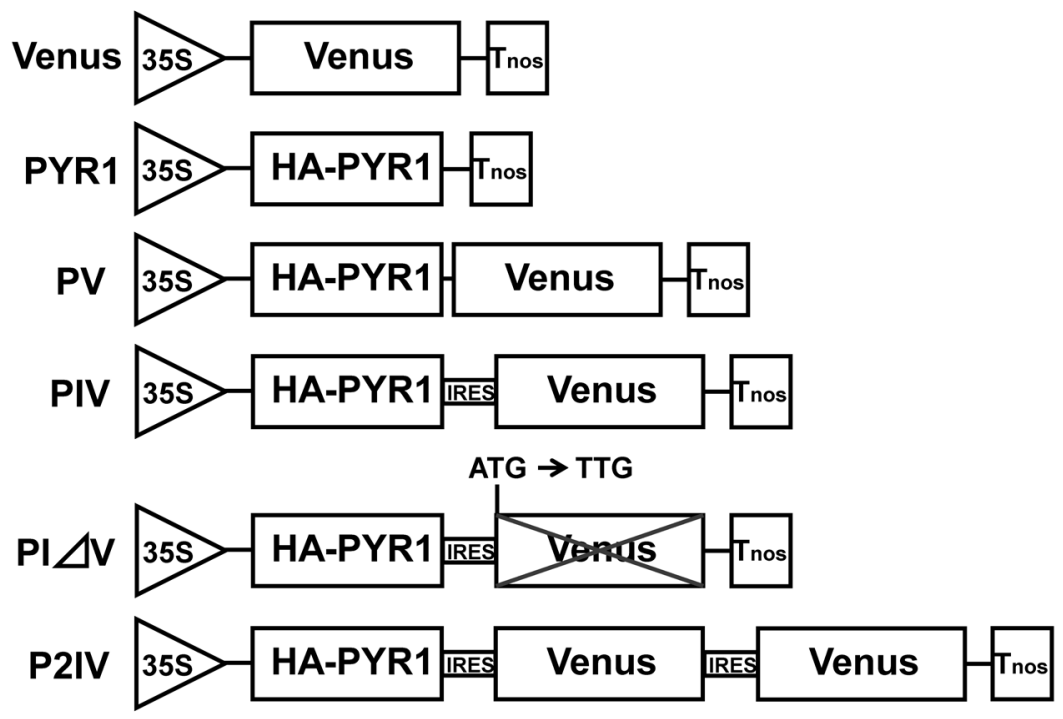

B

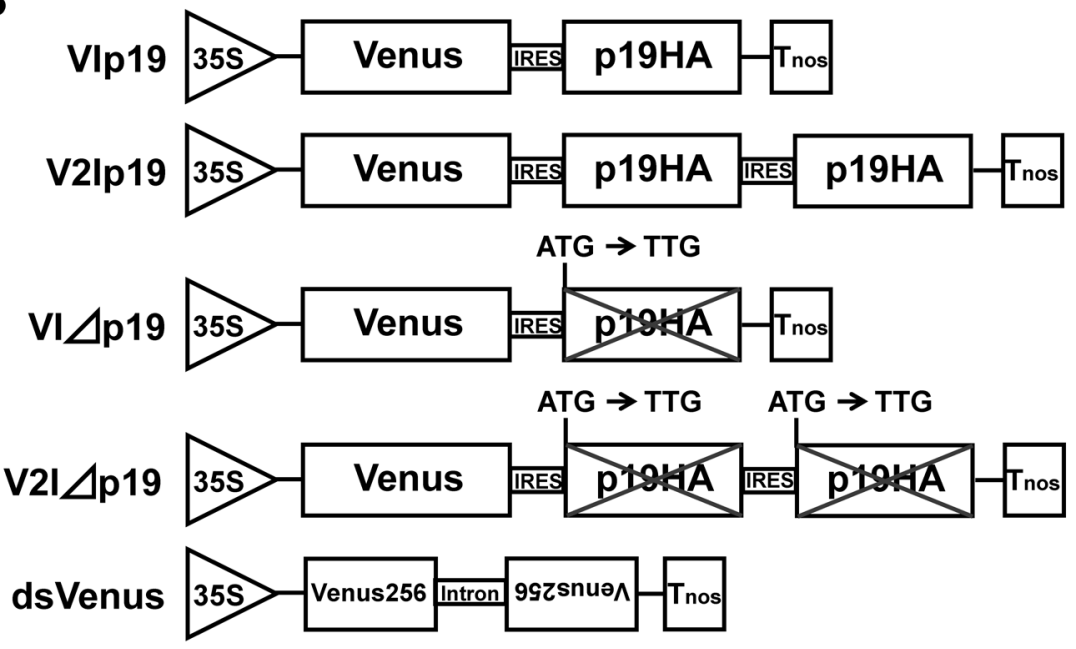

Figure 1. Schematic representation of constructs used for Agrobacterium-mediated transient expression assay in Nicotiana benthamiana. 35S: $35 S$ promoter from the Cauliflower mosaic virus; Venus: Venus gene coding sequence; Tnos: Agrobacterium nopaline synthase terminator; HA-PYR1: HAtagged Arabidopsis abscisic acid receptor coding sequence; IRES: cr-TMV CP internal ribosome entry site; p19HA: HA-tagged p19-HA fusion gene coding sequence. In PI $\Delta$ V, VI $\Delta$ p 19 and V2I $\Delta$ p 19 the start codon of Venus or p19HA is replaced with TTG. Each fragment was inserted into the pSK plant transformation vector.

\section{Materials and methods}

\section{Transgene constructs}

The Venus gene (V), IRES sequences (I) and the p19$H A$ fusion gene (p19) used in this report were derived from the Venus-pTA7002 ${ }^{\mathrm{GW}}$ (Fujioka et al. 2007), yy376 (Yamamoto et al. 2003) and pBICp19HA (Kurihara et al. 2007; Takeda et al. 2002) plasmids, respectively. The HAtagged abscisic acid receptor (HA-PYR1, P) sequence was amplified by polymerase chain reaction (PCR) from an Arabidopsis cDNA library using the following primers: 5' ATC TAG ATG TAT CCA TAT GAT GTA CCA GAT TAT GCT ATG CCT TCG GAG TTA ACA CCA G-3' and 5' -AAA AAA CTA GTT CAC GTC ACC TGA GAA CCA CTT CC- $3^{\prime}$. The constructs depicted in Figure 1 were constructed by PCR amplification, restriction endonuclease digestion and ligation of selected fragments into a pSK1 vector (Kojima et al. 1999). To construct double-stranded Venus (dsVenus), a partial Venus fragment (256 nt) was amplified using the 5' -CAC CGC TGG ACG GCG ACG TAA ACG-3' and 5' ACT AGT GTC CTT GAA GAA GAT GGT GC-3' primers, subcloned into the pENTR/D-TOPO vector and then transferred into the pBI-sense, antisense Gateway vector (Nemoto et al. 2009) using LR clonase II (Invitrogen, Life Technologies, Tokyo, Japan).

Transient expression assay

Agrobacterium strain GV3101 was transformed with the 
constructs described in Figure 1 and cultivated for $20 \mathrm{~h}$ at $29^{\circ} \mathrm{C}$ in LB medium containing $50 \mu \mathrm{g} / \mathrm{ml}$ kanamycin. Agrobacteria cells were pelleted by centrifugation, resuspended and diluted in infiltration medium (10 mM MES, $\mathrm{pH} 5.8,10 \mathrm{mM} \mathrm{MgCl}_{2}$ and $100 \mu \mathrm{M}$ acetosyringone) to an $\mathrm{OD}_{600 \mathrm{~nm}}$ of 0.5 and incubated at room temperature for $2 \mathrm{~h}$. The Agrobacterium solution was inoculated by syringe into holes in the leaves of one-monthold Nicotiana benthamiana plants. Leaves were used for analysis three days after inoculation for all experiments, except those depicted in Figure 3D and E, in which leaves were used six days after inoculation.

\section{Protein analysis}

Leaves were ground to a fine powder in liquid nitrogen and mixed with an equal volume of $2 \times$ SDS sample buffer $(0.125 \mathrm{M}$ Tris- $\mathrm{HCl}, \mathrm{pH}$ 6.8, 4\% SDS, $20 \%$ glycerol, $10 \%$ 2-mercaptoethanol, $0.01 \%$ bromophenol blue). The homogenized sample was incubated at $95^{\circ} \mathrm{C}$ for $5 \mathrm{~min}$ and centrifuged for $5 \mathrm{~min}$ at $14,000 \mathrm{rpm}$. Equal volumes of each supernatant were loaded into wells of a $12 \%$ SDS-PAGE gel and subjected to electrophoresis at $4 \mathrm{~V} / \mathrm{cm}$. Proteins separated by the gel were transferred to an Immobilon-P PVDF membrane (Millipore, MA USA) using a wet-type blotting apparatus followed by incubation in TBE blocking solution with 5\% skimmed milk for $1 \mathrm{~h}$. HA-PYR1 and p19-HA proteins were detected using an anti-HA monoclonal antibody conjugated with peroxidase (Roche, Basel Switzerland). Venus protein was detected using the anti-Green Fluorescent Protein (GFP) polyclonal antibody (Abcam, Cambridge, UK) and the antirabbit IgG peroxidase-linked antibody (GE Healthcare, Little Chalfont, UK) as the primary and secondary antibodies, respectively. Bands were visualized using the enhanced chemiluminescence (ECL) prime reagent (GE Healthcare) according to manufacturer's instructions.

\section{$R T-P C R$ analysis}

Total RNA was extracted from leaves of Nicotiana benthamiana using Trizol reagent (Invitrogen) according to manufacturer's instructions. After digestion with DNase I (Takara, Kyoto, Japan), samples were subjected to phenol:chloroform extraction and ethanol precipitation. One microgram of total RNA was reverse-transcribed using the PrimeScript II 1st Strand cDNA Synthesis Kit (Takara). Fragments were amplified from the cDNA using the following primers: 5'-CAG CCA CAA CGT CTA TAT CACC-3' and 5' GAA CTC CAG CAG GAC CAT GTG-3' for Venus, 5' -CTG ACG AAT TAC AAA TCC GTT AC- $3^{\prime}$ and $5^{\prime}$-CGT CAC CTG AGA ACC ACT TCC-3' for PYR1, and 5'-AGA CCA ATG CGG AGC ATA TAC G-3' and $5^{\prime}$-ACC TGC CTG AAA CCGAACTG-3' for the hygromycin phosphotransferase gene $(H P T)$. The HPT gene is located in the T-DNA region of the pSK1 vector [16]. PCR products were size fractionated on a $1.5 \%$ agarose TBE gel, stained with ethidium bromide and visualized under ultraviolet light. Signal intensities were calculated using ImageJ software (http://rsb.info.nih.gov/ij/).
The significant difference in Figure 3E was statistically defined by a Student's $t$-test $(p<0.05)$ using data from four biological replicates.

\section{Northern blot analysis}

Twenty microgram of total RNA was resolved with electrophoresis on a denaturing $15 \%$ polyacrylamide gel containing $7 \mathrm{M}$ urea in $0.5 \mathrm{x}$ TBE buffer and electroblotted onto Hybond-N+ membrane (GE Healthcare, Little Chalfont, $\mathrm{UK}$ ) in $0.5 \mathrm{x}$ TBE buffer. Membrane was soaked in 2x SSC for $5 \mathrm{~min}$, crosslinked by UV exposure $\left(70,000 \mathrm{~J} / \mathrm{cm}^{2}\right)$ and rinsed in $0.5 x$ SSC. Radiolabeled Venus DNA probes were constructed by random priming method using $\alpha-{ }^{32} \mathrm{P}$-dCTP (PerkinElmer, Inc., MA, USA) and Rediprime II DNA labelling system (GE Healthcare). Hybridization was performed at $40^{\circ} \mathrm{C}$ using Perfect Hyb plus hybridization buffer (Sigma). Membrane was washed two times with $2 \mathrm{x}$ SSC, $0.1 \%$ SDS for $10 \mathrm{~min}$ and three times with $0.5 \mathrm{x}$ SSC, $0.1 \%$ SDS for $15 \mathrm{~min}$ at $50^{\circ} \mathrm{C}$. Radioactive signals were detected using BAS2500 imager (Fujifilm, Tokyo, Japan).

\section{Fluorescent microscopy}

Venus fluorescence in transgenic leaves was visualized using a Zeiss Confocal microscope (Zeiss, LSM510META, Oberkochen Germany). Photos presented in the same figure were taken using the same exposure time.

\section{Results and discussion}

\section{A double IRES cassette enhances IRES-mediated expression}

To optimize cr-TMV CP IRES-mediated gene expression in plants, polycistronic constructs comprised of $H A$ PYR1 and one or two Venus genes with the IRES sequence (Figure 1A) were transiently expressed in Nicotiana benthamiana. We selected PYR1 gene encoding an abscisic acid receptor as the $5^{\prime}$ proximal gene, because HA-PYR1 protein was previously efficiently expressed in this system (Nishimura et al. 2009). We also selected Venus gene encoding a variant of GFP as a reporter of IRES-mediated expression. There is a tendency for lower accumulation of mRNA as the mRNA length increases (Figures 1A, 2A). The amount of PYR1 protein was almost same in plants transfected with PYR1, PIV and P2IV, although PYR1 mRNA level differed among these plants (Figure 2). The amount of Venus protein became lower in PIV-transfected plant in which Venus was expressed polycistronically than in plant in which Venus was expressed alone (Figure 2B). To rule out the possibility of any leaky translation of the upstream PYR1 gene into the Venus gene, we made a PYR1-Venus construct without the IRES sequence (PV) (Figure 1). We observed hardly any Venus protein with this construct, while we observed clear accumulation of Venus protein with PIV (Figure 2C). When two Venus genes with IRESs were attached in tandem (P2IV) we observed 
A

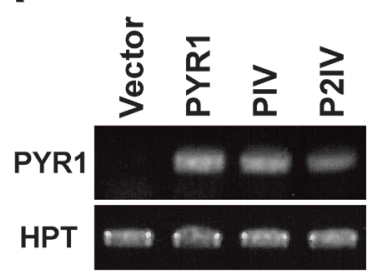

C

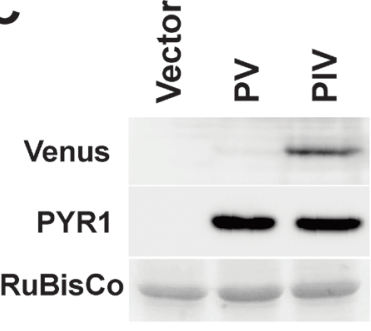

B

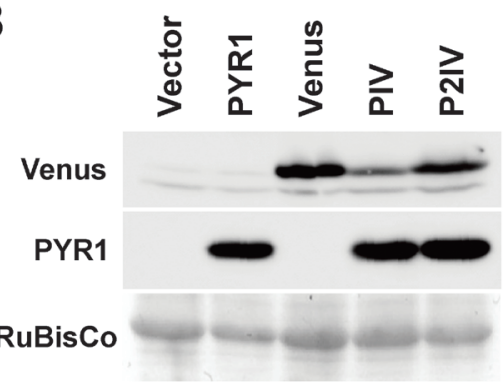

D

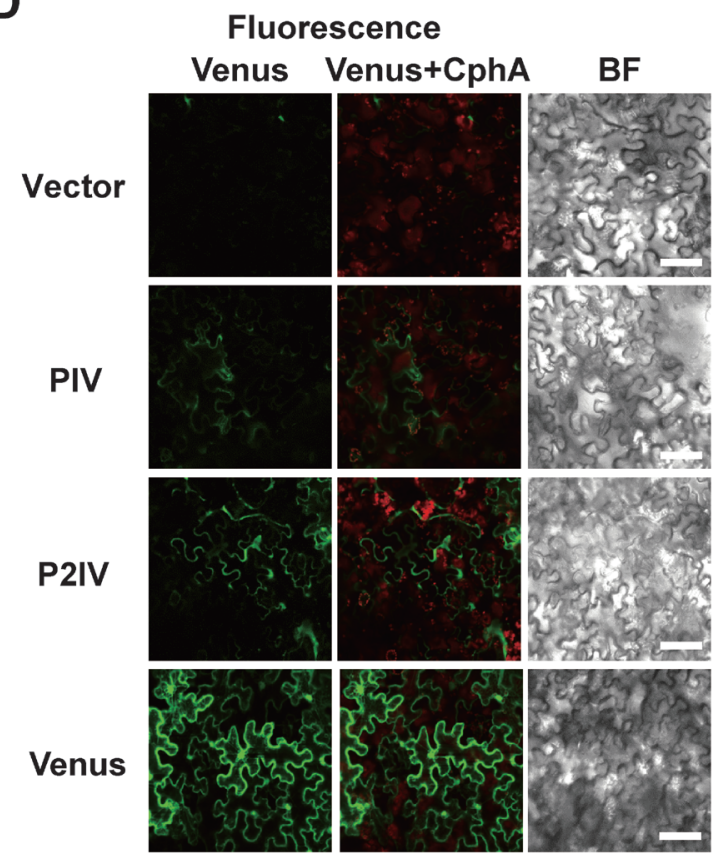

Figure 2. Evaluation of the double IRES cassette system. (A) Quantitative RT-PCR analysis of mRNAs accumulating in leaves infected with each construct. The HPT gene in the pSK1 vector was used as a control. (B) and (C) western blot analysis of Venus and HA-tagged PYR1 transient expression. Ribulose-1,5-bisphospahte carboxylase (RuBisCo) protein was used as a loading control. (D) Fluorescent image of Venus protein expressed in leaves infected with PIV, P2IV and Venus constructs. Vector without any insert was used as the control. Venus: Fluorescent image of Venus. Venus+CphA: Fluorescent image of Venus and chlorophyll A (CphA). BF: Image of bright field. Venus fluorescence is green. CphA autofluorescence is red.

much higher accumulation of Venus protein than in PIV (Figure 2B). These results were also confirmed with in vivo fluorescence (Figure 2D). P2IV transfected tobacco leaves gave clear Venus fluorescence.

A similar attempt using multiple IRES cassettes to express an internal gene for an animal system demonstrated that IRES-driven expression was proportional to the number of IRES cassettes in the polycistronic vector (Bouabe et al. 2008). Our results indicate that, as in the animal system, IRES-mediated expression can be used in a copy-dependent manner in plants.

\section{Application of the double IRES system for suppression of RNA silencing}

The double IRES cassette system was used to examine suppression of RNA silencing mediated by siRNAs.
Constructs were generated that contained one Venus gene at the $5^{\prime}$ end followed by one or two IRES cassettes with the $p 19$ gene, a Tombusvirus suppressor of RNA silencing (VIp19 and V2Ip19) (Figure 1B). The dsVenus construct was made enabling transcription of doublestranded RNAs from a $256 \mathrm{nt}$ Venus sequence to induce RNA silencing of Venus, allowing us to examine whether IRES-mediated p19 protein protects its own mRNA (VIp19 or V2Ip19) from Venus siRNAs. Controls for VIp19 and V2Ip19 were generated by replacing the start codon (ATG) of the $p 19$ gene with a TTG codon to depress p19 translation (VI $\Delta \mathrm{p} 19$ and V2I $\Delta$ p 19 ). As a result of this modification, the longer V2Ip19 and V2I $\Delta$ p 19 mRNAs were accumulated at lower amounts than the shorter VIp 19 and VI $\Delta$ p 19 mRNAs (Figure $3 \mathrm{~A}$ ). Western blot analysis of these constructs in the Nicotiana benthamiana transient expression assay indicated that the 
A

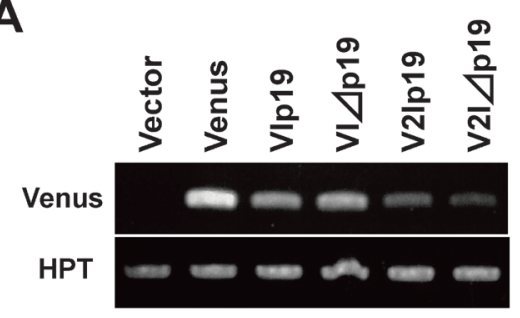

B

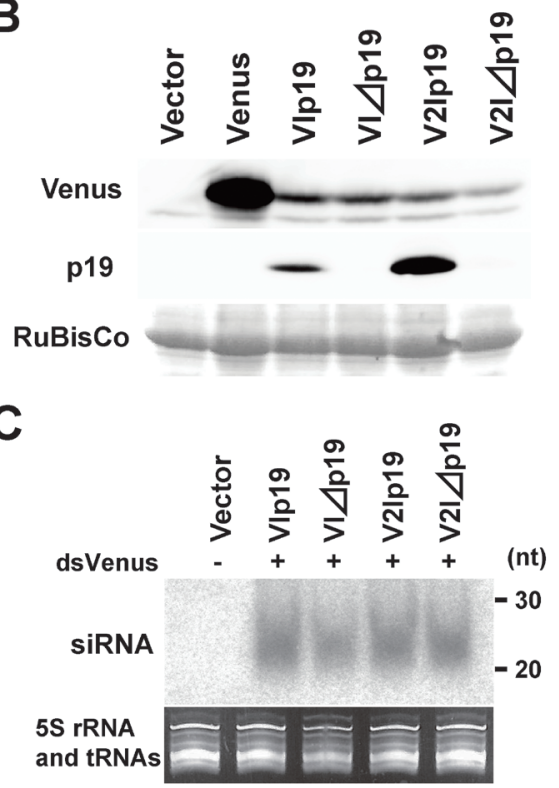

$\mathbf{F}$

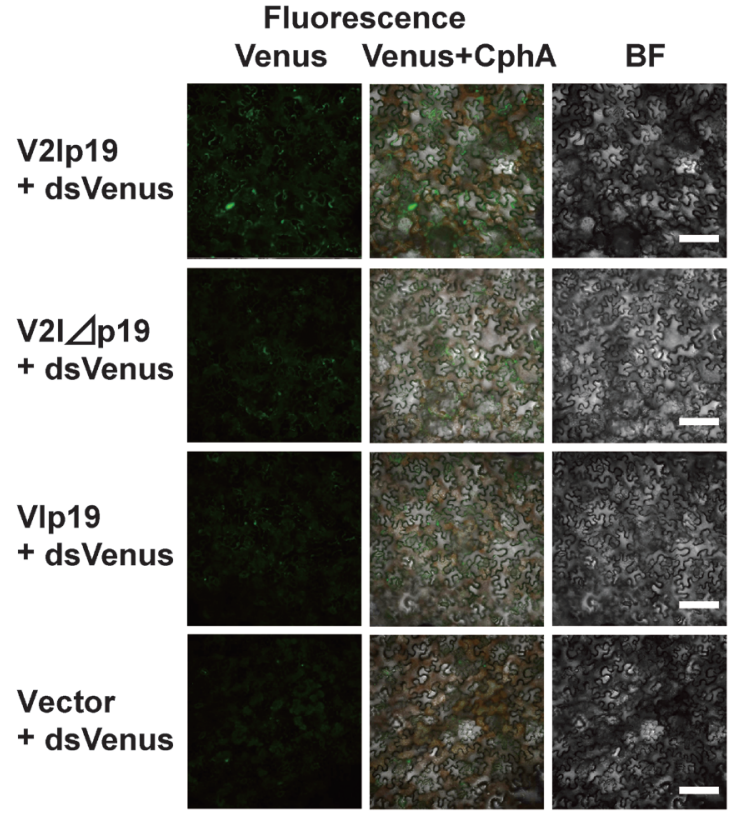

D

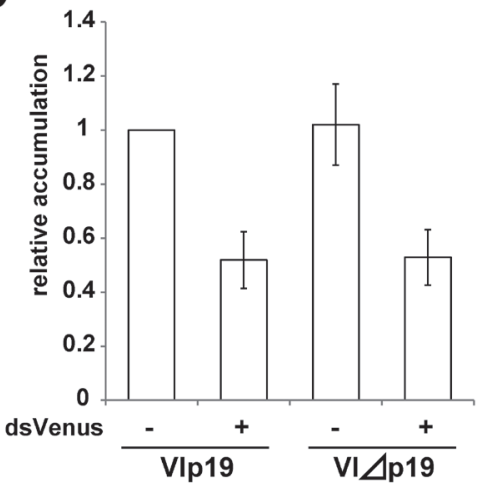

$\mathbf{E}$

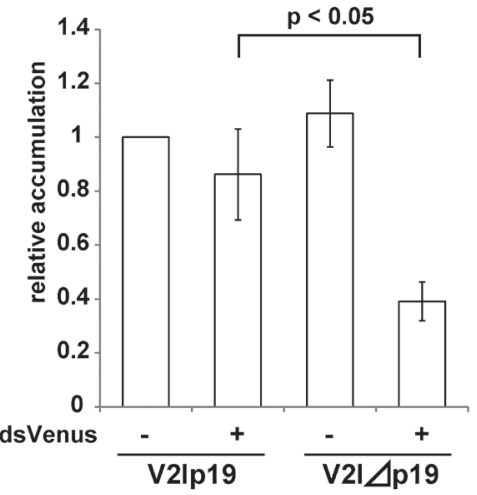

Figure 3. A double IRES cassette system for suppression of RNA silencing. (A) Quantitative RT-PCR analysis of mRNA accumulating in leaves infected with each construct. The HPT gene in the pSK1 vector was used as a control. (B) Western blot analysis of Venus and p19 proteins in leaves infected with each construct; RuBisCo protein was used as a loading control. (C) Northern blot analysis of Venus siRNAs arisen from dsVenus. The image of 5S rRNA and tRNAs was use as a loading control. (D) Quantitative RT-PCR analysis of VIp19 and VI $\Delta \mathrm{p} 19$ in the presence or absence of co-inoculated dsVenus transcript. (E) Quantitative RT-PCR analysis of V2Ip19 and V2I $\Delta$ p19 in the presence or absence of co-inoculated dsVenus transcript. In C and D the vertical axes indicate the relative expression of Venus normalized to HPT expression. In each graph expression of Venus in VIp19 and V2Ip19 without dsVenus was adjusted to "1". Four biological replicates were performed. Significant difference was defined by a Student's $t$ test. (F) Fluorescent image of leaves infected with the vector, VIp19, V2Ip19 and V2I $\Delta$ p19 constructs co-inoculated with dsVenus. Venus fluorescence is green. CphA: chlorophyll A; BF: bright field. 
amount of p19 protein from V2Ip19 was much higher than that from VIp19 (Figure 3B).

Northern blot analysis showed that Venus siRNAs arisen from the dsVenus accumulated at comparable level irrespective of co-infection with VIp19, VI $\Delta$ p 19 , V2Ip19 or V2I $\Delta$ p19, comfirming induction of RNA silencing by the dsVenus (Figure 3C). Quantitative RTPCR analysis indicated that co-infection of the dsVenus construct decreased Venus accumulation in VIp19 and VI $\Delta$ p19 to half of that without dsVenus (Figure 3D). This result may indicate that neither VIp19 nor VI $\Delta$ p 19 mRNAs were protected from RNA silencing. In contrast, co-infection of dsVenus decreased the accumulation of Venus in both V2I $\Delta$ p 19 and V2Ip19 but the reduction was less in V2Ip19 than in V2I $\Delta$ p19 (Figure 3E). We confirmed these results by microscopic examination. After co-infection with dsVenus, fluorescence of Venus from V2Ip19 was clearly observed while weaker fluorescence was seen in VIp19 (Figure 3F). When p19 protein translation was inhibited by replacing the first codon with TTG (V2I $\Delta \mathrm{p} 19)$ we could observe very low fluorescence at the same level as that of VIp19 when coinfected with dsVenus. This result indicated polycistronic expression and translation of $\mathrm{p} 19$ protein suppressed its own mRNA gene silencing depending on the dose of $\mathrm{p} 19$.

As shown in Figure 3B, the amount of p19 protein in VIp19 is considerably lower compared with that in V2Ip19. Taking into account the lower mRNA level, double p19 with an IRES can produce more than double the amount of p19 protein than that of a single p19 (Figure 3B). We speculate that the amount of $\mathrm{p} 19$ protein produced from VIp19 is not enough to bind the siRNAs produced from dsVenus. In contrast, double p19 with an IRES (V2Ip19) can efficiently suppress siRNAs. These results indicate that supplying p19 with a double IRES is effective in protecting the mRNA from silencing.

\section{Evaluation of the double IRES cassette system}

PYR1 accumulation from the $5^{\prime}$ proximal gene was comparable in PYR1, PIV and P2IV (Figure 2B), while Venus accumulation from the $5^{\prime}$ proximal gene was different in Venus, VIp19 and V2Ip19 (Figure 3B). To rule out the possibility that translation of the $3^{\prime}$ gene, Venus, of PIV positively affects translation of the $5^{\prime}$ gene, PYR1, we compared protein accumulation from PYR1, PIV and PI $\Delta \mathrm{V}$ that does not possess the start codon of Venus (Figure 1A). We found that PYR1 accumulation was not significantly different between PIV and PI $\Delta \mathrm{V}$, regardless of whether the Venus gene was translated or not (Figure 4). This result indicates that translation of the $3^{\prime}$ gene does not affect translation of the $5^{\prime}$ gene in polycistronic expression.

The stability and efficiency of cap-dependent translation of PYR1 from PIV and P2IV, and of Venus from VIp19 and V2Ip19 were not established. Generally,

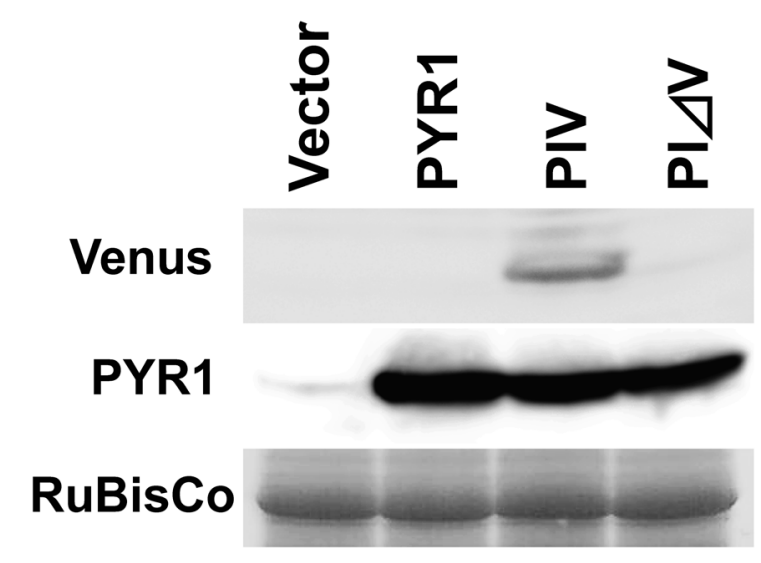

Figure 4. Western blot analysis of Venus and HA-tagged PYR1 transient expression from vector, PYR1, PIV and PI $\Delta$ V. RuBisCo protein was used as a loading control.

protein accumulation seems to be proportional to the mRNA level, which is dependent on mRNA length when the same promoter is used. As shown in Figure 3A and $\mathrm{B}$, the longer V2Ip19 mRNAs were expressed at lower levels than the shorter Venus and VIp19 mRNAs, and accumulation of Venus protein was proportional to the mRNA levels. On the other hand, despite the fact that the P2IV mRNAs were longer they were expressed at lower levels than the shorter PYR1 and PIV mRNAs (Figure 2A), resulting in PYR1 accumulation that was comparable (Figure $2 \mathrm{~B}$ ), but not proportional, to mRNA levels. These results indicate that comparable amounts of PYR1 proteins in PYR1, PIV and P2IV were probably due to protein stability, but not due to efficiency of capdependent translation.

As described above, the IRES-mediated polycistronic expression system possesses some weak points. One of them is that protein accumulation from the $5^{\prime}$ proximal gene might be lower than when it is expressed alone. This negative effect sometimes may limit opportunity to use the IRES system. Most possible usage is that we check expression level of a transgene in stable transformants by IRES-mediated co-expression of a reporter gene. In such cases, the $5^{\prime}$ proximal transgenes are limited to those of which functions are active independent of amount of the products.

\section{mRNA self-protection using IRES is useful}

We first established that increasing the number of IRESmediated gene cassettes increases corresponding gene expression in our Nicotiana benthamiana transient expression assay (Figure 2). As an application of this system, we also demonstrated that expression of the Tombusvirus p19 protein, an RNA silencing suppressor, from a double IRES cassette-but not single cassettesuppressed RNA silencing of its own mRNA (Figure 3). This is the first report of a mRNA self-protection system from RNA silencing by IRES-mediated expression of a 
viral suppressor gene in plants. Here, we propose that it is beneficial to use polycistronic expression of RNA silencing suppressors as protection for a plant's own mRNA.

In plants the presence of multiple copies of transgenic sequences, such as the $35 \mathrm{~S}$ promoter, sometimes induces gene silencing of the transgenes (Daxinger et al. 2008; Velten et al. 2012; Weinhold et al. 2013). This silencing trap might be avoided when several transgenes are required by using IRES-mediated gene expression of polycistronic constructs. Furthermore, the use of the IRES system enables the synchronous expression of multiple genes using a single promoter. Thus, the double IRES cassette system provides a useful tool for expressing multiple genes polycistronically in plants.

\section{Acknowledgements}

This work was supported by a Grant-in-Aid for Young Scientists (B) from the Japan Society for the Promotion of Science to Y. K. (No. 25871119).

\section{References}

Baulcombe D (2004) RNA silencing in plants. Nature 431: 356-363

Bouabe H, Fassler R, Heesemann J (2008) Improvement of reporter activity by IRES-mediated polycistronic reporter system. Nucleic Acids Res 36: e28

Daxinger L, Hunter B, Sheikh M, Jauvion V, Gasciolli V, Vaucheret H, Matzke M, Furner I (2008) Unexpected silencing effects from T-DNA tags in Arabidopsis. Trends Plant Sci 13: 4-6

Fujioka Y, Utsumi M, Ohba Y, Watanabe Y (2007) Location of a possible miRNA processing site in $\mathrm{SmD} 3 / \mathrm{SmB}$ nuclear bodies in Arabidopsis. Plant Cell Physiol 48: 1243-1253

Ha S-H, Liang YS, Jung H, Ahn M-J, Suh S-C, Kweon S-J, Kim D-H, Kim Y-M, Kim J-K (2010) Application of two bicistronic systems involving $2 \mathrm{~A}$ and IRES sequences to the biosynthesis of carotenoids in rice endosperm. Plant Biotechnol J 8: 928-938

Jang SK, Krausslich HG, Nicklin MJ, Duke GM, Palmenberg AC, Wimmer E (1988) A segment of the 5 '-nontranslated region of encephalomyocarditis virus RNA directs internal entry of ribosomes during in vitro translation. J Virol 62: 2636-2643

Kojima S, Banno H, Yoshioka Y, Oka A, Machida C, Machida Y (1999) A binary vector plasmid for gene expression in plant cells that is stably maintained in Agrobacterium cells. DNA Res 6: $407-410$
Kurihara Y, Inaba N, Kutsuna N, Takeda A, Tagami Y, Watanabe Y (2007) The binding of Tobamovirus replication protein with small RNA duplexes. J Gen Virol 88: 2347-2352

Nemoto K, Hara M, Suzuki M, Seki H, Muranaka T, Mano Y (2009) The NtAMI1 gene functions in cell division of tobacco BY-2 cells in the presence of indole-3-acetamide. FEBS Lett 583: 487-492

Nishimura N, Hitomi K, Arvai AS, Rambo RP, Hitomi C, Cutler SR, Schroeder JI, Getzoff ED (2009) Structural mechanism of abscisic acid binding and signaling by dimeric PYR1. Science 326: 1373-1379

Pelletier J, Sonenberg N (1988) Internal initiation of translation of eukaryotic mRNA directed by a sequence derived from poliovirus RNA. Nature 334: 320-325

Pumplin N, Voinnet O (2013) RNA silencing suppression by plant pathogens: Defense, counter-defense and counter-counterdefense. Nat Rev Microbiol 11: 745-760

Skulachev MV, Ivanov PA, Karpova OV, Rodionova NP, Dorokhov YL, Atabekov JG (1999) Internal initiation of translation directed by the 5 '-untranslated region of the Tobamovirus subgenomic RNA I $\mathrm{I}_{2}$. Virology 263: 139-154

Takeda A, Sugiyama K, Nagano H, Mori M, Kaido M, Mise K, Tsuda S, Okuno T (2002) Identification of a novel RNA silencing suppressor, NSs protein of Tomato spotted wilt virus. FEBS Lett 532: 75-79

Thompson SR (2012) Tricks an IRES uses to enslave ribosomes. Trends Microbiol 20: 558-566

Vargason JM, Szittya G, Burgyan J, Hall TMT (2003) Size selective recognition of siRNA by an RNA silencing suppressor. Cell 115: 799-811

Velten J, Cakir C, Youn E, Chen J, Cazzonelli CI (2012) Transgene silencing and transgene-derived siRNA production in tobacco plants homozygous for an introduced AtMYB90 construct. PLoS ONE 7: e30141

Voinnet O, Pinto VM, Baulcombe DC (1999) Suppression of gene silencing: a general strategy used by diverse DNA and RNA viruses of plants. Proc Natl Acad Sci USA 96: 14147-14152

Voinnet O, Rivas S, Mestre P, Baulcombe DC (2003) An enhanced transient expression system in plants based on suppression of gene silencing by the 19 protein of tomato bushy stunt virus. Plant J 33: 949-956

Weinhold A, Kallenbach M, Baldwin IT (2013) Progressive 35S promoter methylation increases rapidly during vegetative development in transgenic Nicotiana attenuata plants. BMC Plant Biol 13: 99

Yamamoto YY, Tsuhara Y, Gohda K, Suzuki K, Matsui M (2003) Gene trapping of the Arabidopsis genome with a firefly luciferase reporter. Plant J 35: 273-283 